\title{
Effects of Different Types of Progress Indicators on Drop-Out Rates in Web Surveys
}

\author{
Uwe Matzat, Chris Snijders, and Wouter van der Horst
}

Eindhoven University of Technology, The Netherlands

\begin{abstract}
The present study analyzes whether and how different types of progress indicators affect the tendency of respondents to continue filling out a web survey, focusing on whether the progress indicators' effects depend on the position of the respondent in the questionnaire. Using a sample of 2460 respondents of a Dutch online access panel, we analyze three kinds of progress indicators (linear, fast-then-slow, slow-then-fast, and a control condition) using survival analysis. The results show that the effect of the indicators on the completion rate is either negative or nonexistent, depending on the questionnaire length. Moreover, the effect of an indicator does not depend on the position of the respondent in the answering process. We interpret our findings in terms of the implicit narrative between survey designer and respondent.
\end{abstract}

Keywords: web surveys, progress indicator, completion rates, applying survival analysis

\section{Introduction}

Web surveys are often used in commercial and scientific research. A high response rate is crucial for reducing the potential selectivity bias of the collected data. Many researchers have proposed that a careful design of the web questionnaire increases the respondents' motivation to continue with the answering procedure (see Dillman, 2000 for an overview). Why a careful design might help is not completely clear. Progress indicators are one possible element of careful design: While in a single-page web survey respondents can easily see how long the questionnaire is, this is not the case in page-by-page web surveys. Since pageby-page web surveys offer other advantages (Couper, 2000), they are, nevertheless, often used and recommended (Bosnjak \& Tuten, 2001; Bandilla \& Bosnjak, 2000; Gräf, 2002). Existing research on progress indicators has yielded mixed results. We tried to gain a better understanding of the implicit interaction between survey designer and respondent by analyzing in more detail the effects that progress indicators have on respondent drop-out.

The study differs from previous research in several ways. First, we emphasized the role of progress indicators as one way in which the survey designer communicates with the respondent. Because in an Internet survey there is no face-toface contact between designer and respondent, all communication goes through the design and lay-out of the survey. This may include more than what is actually written. Our assumption is that the survey design and lay-out provide signals about the designer's intentions and create in the respondent a general feeling about what the designer wants as well as how seriously the designer is involved in the survey topic. This is what Schwarz (1998) has called the "narrative approach" in the context of experimental social-psychological research. Tourangeau, Rips, and Rasinksi (2007, pp. 50ff) argue that respondents use this information to draw inferences - correctly or incorrectly- about the designer's intentions. There is evidence suggesting that this also happens in web surveys (Smyth, Dillman, Christian, \& Stern, 2006; Tourangeau et al., 2007). This study elaborates on this idea for the example of progress indicators.

Second, we analyzed dropout rates throughout the survey instead of just the completion rates. In many web surveys, including ours, the data are written to the server "page-wise," so treating only the respondents who have completed the questionnaire as the ones who deliver data is, therefore, more restrictive than necessary. Survival analysis allows us to model the probability of reaching the next page as dependent on the kind of progress bar and several covariates such as the length of the questionnaire and the elapsed time, allowing more extensive use of online survey data and more appropriate and direct testing of different ideas about how progress indicators might affect dropout in online surveys.

\section{Theoretical Background}

Since the end of the 1990s researchers have expected that the existence of progress indicators would increase the mo- 
tivation of respondents to continue filling out a web questionnaire (Dillman, 2000). Several reasons have been mentioned for this alleged positive effect. One reason could be that it shows the care-intensity of the questionnaire's designer, which might spark positive reciprocity in respondents. Progress indicators also give respondents precise information about the burden or opportunity costs of completing the survey so that a respondent can balance costs and rewards of participation (Crawford, Couper, \& Lamias, 2001; Heerwegh, 2004). This prevents that respondents stop answering the questions because they expect the survey to be longer than it really is.

This line of reasoning starts from the assumption that at some point respondents get bored and want to quit, and that the designer communicates the message "you are almost done, hang in there" implicitly through the use of the progress indicator (see, e.g., Heerwegh, 2004, p. 5). Initial studies did not find much support for such positive expectations of the effect of progress indicators. Couper, Traugott, and Lamias (2001) found a nonsignificant 3.5\% increase in the completion rate in a student sample. They attributed this to the fact that the progress indicator increased the download time of the questionnaire, which might have counteracted the effect of the indicator. In a subsequent experiment, Crawford et al. (2001) controlled for the download time and then found that the progress indicator decreased the completion rate. The survey they used contained a number of time-consuming open questions in the first half of the questionnaire. The displayed progress, however, was based on the number of questions, disregarding the amount of time needed to answer them. In post hoc analyses, the authors realized that the progress indicator led to an exaggerated perceived time burden for the respondent. This finding has led to the study of the effects of different types of progress indicators on drop-out rates. Conrad, Couper, Tourangeau, and Peytchev (2005) distinguished three types of indicators. The first type, the constant speed or linear indicator, displayed the progress of a respondent as a linear function of the proportion of completed survey pages (which was almost equivalent to the number of completed questions). The second type of progress indicator, the fast-then-slow or degressive indicator, exaggerated the respondent's progress in the first half of the survey. As a consequence, the progress in the second half of the survey is then slower than actual. The third type of indicator, the slow-then-fast or progressive indicator, underestimated the progress of the respondent in the first half of the survey.

Completion rates for three respondent groups were compared to the completion rate of respondents who completed the same questionnaire without any visible progress indicator. Using respondents from two commercial survey panels, the authors found that the slow-then-fast progress indicator resulted in a reduction in the completion rate when compared with the completion rates of the other three groups. The completion rates among the other three groups of respondents did not differ significantly.
The authors interpreted the findings as supportive for the hypothesis that the first impressions of respondents - the behavior of the progress indicator in the beginning of the questionnaire - affect the decision to drop out, and that the behavior of the indicator in the second half of the survey does not matter as much. Stated in terms of the implicit narrative between designer and respondent, they claim that respondents draw inferences about their time burden at the beginning of the questionnaire. Heerwegh (2004) and Heerwegh and Loosveldt (2006) also compared the answering behavior of respondents who were confronted with a fast-then-slow progress indicator with the answering behavior of respondents who had no progress indicator. As in Conrad et al. (2005), they did not find a significant increase in the completion rate. The fast-then-slow indicator had a lower missing data rate and respondents were significantly less likely to indicate in an open question that the survey was too long. Heerwegh (2004) regarded these findings as being in line with the "first impressions matter" hypothesis of Conrad et al. (2005). An alternative explanation exists that might fit these results as well: The progress indicator is of importance at the point in the survey where the respondent wants to be reassured as to how long the rest of the survey is going to take. If respondents typically want to know this after or while completing tedious and/or complicated questions, then what one would need to have is a progress indicator that is going fast precisely at those moments.

Böhme (2003) compared the three types of progress indicators and a control condition without a progress indicator. He found that the completion rate in the slow-then-fast progress indicator condition was significantly lower than the completion rate in the fast-then-slow progress indicator condition. However, none of the completion rates of respondents in any of the three indicator conditions differed significantly from the completion rate of the respondents in the control condition. Two other findings were remarkable. First, respondents who scored high on computer literacy needed less time to fill out the survey. In itself that is not surprising, but this effect was significantly stronger under the condition of a fast-then-slow progress indicator. The type of indicator seemed to have interacted with the respondent's experience. Second, self-reported satisfaction with the whole questionnaire among respondents in the fast-then-slow indicator condition, measured at the end of the questionnaire, was slightly lower than the satisfaction of respondents in the slow-then-fast indicator condition. Böhme (2003) attributes this to the fact that in the second half of the questionnaire, the slow-then-fast indicator progresses quicker than the fast-then-slow progress indicator. At the end of the questionnaire, respondents evaluate the fast-then-slow indicator worse because the number of questions in the second half turns out to be higher than the respondent expected. If additional research supports this view, then this would not be in accordance with the hypothesis of Conrad et al. (2005).

There are other reasons to believe that progress indi- 
cators could affect drop-out rates in different ways. In this light, it is interesting to reevaluate the suggestion by Heerwegh (2004, p. 5), mentioned above, about why progress indicators might increase completion rates. Implicit is the assumption that in the beginning of a survey the respondents are motivated enough to answer questions but that this motivation deteriorates over the course of the survey. If that is the case, the usefulness of a progress indicator will tend to surface toward the end of the survey (or, at least, not in the beginning), where those respondents who are tempted to quit are perhaps persuaded to finish the last couple of questions when they see that the end is near. Stated in terms of the narrative between designer and respondent, respondents could draw inferences about their time burden when their motivation deteriorates, usually in later parts of the questionnaire.

Hence, there are two points of view about how progress indicators affect a respondent's motivation to continue with a web survey. Both assume that respondents draw inferences about their time burden. One is that the first impression of an indicator on the respondent influences the motivation to continue with the survey and sets the tone for the rest of the survey. The other is that the indicator's effects surface at the end of a survey and that typically indicators that behave differently at the end of a survey should have different effects. Therefore, the first question of this study is as follows: Do the effects of the different types of progress indicators change during the course of the questionnaire? If it is, indeed, the case that "first impressions matter," as suggested in Conrad et al. (2005), then one should find that the effects of progress indicators are independent of the respondent's position in the survey. Moreover, the use of progress indicators is one way in which the designer communicates with the respondent, and distinguishing between the effects of progress indicators in different parts of the survey will give a more detailed view of the reasons why indicators affect completion rates.

If the effect of a progress indicator is variable, then it is likely that respondents' reactions to the speed of progress cause these effects. This implies that the effect of a progress indicator itself can depend on the length of the questionnaire. A second question is thus whether the effects of the different types of progress indicators depend on the questionnaire length. Effect refers to differences in the completion rate, in the needed time for the completion, and in the respondent's subjective satisfaction with the perceived burden of the survey. A third question is whether there is any effect of the elapsed time. Here we do not have clear expectations. As time progresses, respondents might get fed up and quit or feel that they might as well finish the survey. A fourth question is whether the type of progress indicator moderates the effect of the respondent's computer literacy on completion time and whether the speed of the indicator and the end of the questionnaire affects the respondent's survey sat- isfaction, as Böhme (2003) suggests. Thus, while we cannot always deduce unambiguous effects, we summarize the theoretical background of our study for easier reference in five testable hypotheses.

- Hypothesis 1: Effects of the progress indicators on the respondent's probability to continue are independent of the position in the survey ("first impressions matter").

- Hypothesis 2: Effects of the progress indicators on the respondent's probability to continue vary between the shorter and the longer version of the survey.

- Hypothesis 3: The more time has elapsed since the start of the survey, the higher the respondent's probability to continue.

- Hypothesis 4: The respondent's satisfaction with the survey at the end of the questionnaire is higher for the SLOW-THEN-FAST indicator than for the FASTTHEN-SLOW indicator.

- Hypothesis 5: The negative effect of the respondent's computer literacy on the completion time is stronger for the FAST-THEN-SLOW indicator than for the SLOWTHEN-FAST indicator.

\section{Method}

\section{Procedure}

An invitation to participate in our survey was sent by email to a random sample of 5646 members of a large Dutch commercial 'opt in' access panel. Of these 5646 panel members, 2553 (45.2\%) clicked on the link in the invitation.

The web survey consisted of more than 40 pages with at most 55 (for the short version) or $55+30$ (for the long version) questions, depending on whether the respondent was assigned to the long or short questionnaire condition. The topics on the survey included questions on Internet use, opinions about governmental policies, the social network of the respondent, and the trust the respondent had in people from foreign countries. The invitation announced that it would take about $15-20 \mathrm{~min}$ to complete the questionnaire.

\section{Participants}

From the 2553 persons who clicked the invitation, 2460 (43.6\% of the 5646) actually started the survey by clicking the appropriate button on the introduction page. In the analyses only the 2460 persons who actually started the survey are considered, of which 2197 (89\%) completed the survey. The age of participants varied between 15 and 76 (90\% in the range 17-56), with a mean of 34 and a standard deviation of 12.5. In the last year, participants had completed, on average, 5.1 surveys $(S D=3.1)$ with the panel. 


\section{Design}

We analyzed the effects of two experimental treatments in a $4 \times 2$ (progress indicator times length of survey) betweensubjects design, taking several covariates into account. The first factor, the type of progress indicator, consisted of four conditions to which the respondents were randomly assigned. In the following formulas " $\mathrm{X}$ " indicates the filledout number of questions and " $\mathrm{N}$ " indicates the total number of questions that the respondent had to fill out. In this study, the number of questions is almost equivalent to the number of pages. Questions that might be skipped in later parts of the questionnaire were included in the calculation of the number of questions that had to be answered. For all three progress indicators, this led to small "jumps" in the displayed progression of the indicator if a question was actually filtered out. ${ }^{1}$

1. No progress indicator (NO INDICATOR).

2. A progress indicator with a constant speed of progress, ; the formula used was progress $=100 * \mathrm{X} / \mathrm{N}$ (LINEAR INDICATOR).

3. A fast-then-slow progress indicator that displays a faster progression in the first half of the questionnaire than in the second half; the formula used is progress $=-150 *$ $\left(-1+(1 / 3)^{\wedge} \mathrm{X} / \mathrm{N}\right)($ FAST-THEN-SLOW).

4. A slow-then-fast progress indicator that displays a slower progression in the first half of the questionnaire than in the second half; the formula used is progress $=50 *$ $\left(-1+3^{\wedge} \mathrm{X} / \mathrm{N}\right)($ SLOW-THEN-FAST).

When a progress indicator was offered, it was displayed continuously on each page in the upper right (see Figure 1).

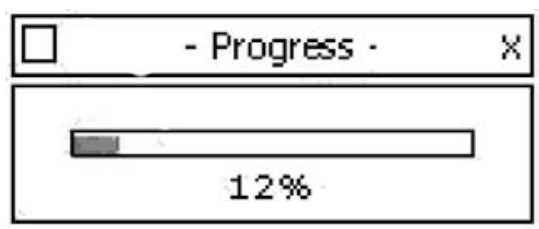

Figure 1. Screenshot of a progress indicator as used in the survey. Note. Although it was not mentioned in the survey, the progress indicators could be made to disappear by the respondent clicking the cross in the top right corner of the graph. None of the respondents, however, used this option.

Respondents were randomly assigned to a short or long survey (dummy: long survey). The long version had one extra page with a matrix consisting of 30 questions regarding the social capital of the respondent (the "position generator"; Lin \& Dumin, 1986). The dependent variables of interest include the drop-out rate at different parts of the survey, self-reports on the perceived satisfaction with the questionnaire, and the time needed to complete the questionnaire.

Drop out was determined by finding the page in the survey where the respondent still answered some questions. Further measurements are reported below:

- Satisfaction: General satisfaction was measured in two ways, at the end of the survey. First, we asked whether respondents were willing to answer a couple of questions regarding the user-friendliness and usability of the survey (dummy variable: allowed extra questions, $M=0.73$ ). Second, five-point Likert scales were used to ask respondents how difficult it was to answer the questions, how user-friendly the questionnaire was, etc. The variable user satisfaction is a factor score (a weighted average), scaled to mean zero and unit variance.

- Age of the respondent: The age of the respondents in years $(M=33.5, S D=12.5)$.

- Elapsed time: The amount of time respondents used up to this specific point, in minutes (a time-varying covariate in the survival analysis).

- Time: Total time spent on the survey $(M=22.2, S D=$ 5.6).

- Survey experience: The number of web surveys that the respondent had completed as a member of the online panel during the last 12 months (experience, $\mathrm{M}=$ $5.1, S D=3.1$ ). In our models we used $\log$ of experience, defined as the natural log of experience +1 . We sometimes used a dummy variable indicating whether the respondent had filled out at least five web surveys during the last 12 months (experienced, $M=0.51$, $S D=0.50)$.

- Computer literacy: A self-reported assessment using a five-point scale: computer literacy (self) $(M=3.3, S D=$ 0.68 ). The second was as an (unweighted) average of how often participants regularly performed 11 computer tasks: computer literacy (tasks) $(M=4.4, S D=4.1)$.

- Checkpoints: We identified 25 checkpoints in the survey that respondents would pass as they completed the survey (dummies per checkpoint: ChP1,..., ChP25 and a linear trend: checkpoint trend).

- Bonus points: The internet panel "pays" the respondents in bonus points, which can be exchanged for certain goods. The amount varied from 50, 100, to 150 and was a random factor in the invitation to the study.

- Type of invitation: Invitation type was also randomly varied to convince potential respondents to participate. There was one invitation with an extensive clarification and an explicitly shown logo of the university, and a short invitation without explanation and without the logo (dummy: short invitation).

1 Such jumps occurred only three times in the whole survey and considered single questions only. This leads to differences in jumps between persons that are minute compared to the jumps one necessarily gets from page to page, and have no influence on our results (we ran all our analyses with separate dummy-variables included). 
Table 1. Completion rate per type of progress indicator (given that respondents have actually started the survey)

\begin{tabular}{lcrl}
\hline & Completed & Total & $\%$ \\
\hline NO INDICATOR & 582 & 634 & $91.8 \%$ \\
LINEAR INDICATOR & 526 & 596 & $88.3 \%$ \\
FAST-THEN-SLOW & 550 & 610 & $90.2 \%$ \\
SLOW-THEN-FAST & 539 & 620 & $86.9 \%$ \\
Total & 2197 & 2460 & $89.3 \%$ \\
\hline
\end{tabular}

Table 2. Completion rates per type of progress indicator, per length of survey

\begin{tabular}{lll}
\hline & Short $(n=1174)$ & Long $(n=1286)$ \\
\hline NO INDICATOR & $93.6 \%$ & $89.5 \%$ \\
LINEAR INDICATOR & $89.6 \%$ & $86.9 \%$ \\
FAST-THEN-SLOW & $88.9 \%$ & $91.0 \%$ \\
SLOW-THEN-FAST & $84.4 \%$ & $89.0 \%$ \\
Total & $89.4 \%$ & $89.2 \%$ \\
\hline
\end{tabular}

\section{Results}

\section{Descriptive Results}

We first compared the raw completion rates per indicator.

Table 1 shows that the highest completion rate occurred in the condition without a progress indicator. The lowest completion rate was found in the case of the slow-then-fast indicator $(86.9 \%)$. The difference between NO INDICATOR and the other conditions was significant at the $p=$ .003 level, $\chi^{2}=8.68, d f=1$. Next, in Table 2 the raw completion rates are compared while distinguishing between the short and the long version of the survey.

In the long version, the highest completion rate occurred for the fast-then-slow progress indicator, although the difference with NO INDICATOR at all is not statistically significant. In addition, both the fast-then-slow and the slowthen-fast indicator perform slightly better in the long version as compared to the short version. Care should be taken with these results: Differences of these magnitudes are marginally significant, at best. For instance, the difference in completion rates between the short and long version of the slow-then-fast indicator was significant at $p=.096, \chi^{2}=$ $2.77, d f=1$. There is evidence that the differences between NO INDICATOR vs. any indicator vary between the short vs. the long version. In the short version, this difference was statistically significant, $p=.002, \chi^{2}=9.22, d f=1$ for NO INDICATOR versus all other indicators, whereas this difference was not significant in the long version, $p=.84$, $\chi^{2}=0.04, d f=1$.

We next considered 24 checkpoints in the survey, which each respondent must pass in order to complete the questionnaire (regardless of the type of the survey they have).
Table 3. Dropout rates at checkpoint 4 per type of progress indicator, per length of survey

\begin{tabular}{llll}
\hline & Short $(n=1174)$ & Long $(n=1286)$ & All \\
\hline NO INDICATOR & $2.8 \%$ & $2.2 \%$ & $2.5 \%$ \\
LINEAR INDICATOR & $4.4 \%$ & $4.7 \%$ & $4.5 \%$ \\
FAST-THEN-SLOW & $4.9 \%$ & $1.9 \%$ & $3.1 \%$ \\
SLOW-THEN-FAST & $4.0 \%$ & $3.5 \%$ & $3.7 \%$ \\
Total & $3.9 \%$ & $3.0 \%$ & $3.5 \%$ \\
\hline
\end{tabular}

Table 4. Overall completion rates per type of progress indicator for experienced an inexperienced respondents

\begin{tabular}{lll}
\hline & Inexp. $(n=1208)$ & Exp. $(n=1252)$ \\
\hline NO INDICATOR & $86.2 \%$ & $97.7 \%$ \\
LINEAR INDICATOR & $78.6 \%$ & $96.8 \%$ \\
FAST-THEN-SLOW & $83.0 \%$ & $97.1 \%$ \\
SLOW-THEN-FAST & $78.9 \%$ & $94.6 \%$ \\
Total & $81.8 \%$ & $96.6 \%$ \\
\hline
\end{tabular}

The zero checkpoint occurred before the indicator was present and the 25th checkpoint was at the end of the survey.

The most remarkable result, besides the 93 dropouts in the beginning, are the 85 dropouts at the fourth checkpoint. This is the point where the initial questions (asking for age, etc.) have been completed and questions about the current situation in the Netherlands begin. Table 3 shows this comparison for both the long and the short survey.

The differences in percentages between the short and the long version were not significant except for the difference between the short and the long version of the fast-then-slow indicator, $p=.04$.

Table 4 shows that the more experienced panel members (at least five completed surveys in the previous year vs. four or less) were much more likely to complete the survey. The difference was almost 15 percentage points, $p<.001$. Given that the completion rates of the experienced respondents were close to $100 \%$, the differences between the progress indicators were negligible. Hence, it seems that the differences between the progress indicators resulted from the differences in behavior of the inexperienced respondents in the panel; the inexperienced respondents showed lower completion rates for all three progress indicators, $p<$ .01 .

\section{Survey-Survival Analysis}

We now turn to the survival analysis. In social science, survival analysis is a standard method to model "time until event" data. Our context is perfectly suited for this technique. We are indeed analyzing survival data: "survey-survival." The dependent variable is whether the respondent reaches the next checkpoint, given that he or she has 
Table 5. Logistic regression analyses on the probability to reach checkpoint $k+1$ in the survey, given that checkpoint $k$ has been reached. Included in the analysis (but not in the table) are separate dummies per checkpoint; coefficients are unstandardized ( $p$-values in parentheses)

\begin{tabular}{|c|c|c|c|c|c|c|c|c|}
\hline & $\mathrm{m} 1$ & $\mathrm{~m} 2$ & $\mathrm{~m} 3$ & $\mathrm{~m} 4$ & $\mathrm{~m} 5$ & m6 & $\mathrm{m} 7$ & $\mathrm{~m} 8$ \\
\hline LINEAR INDICATOR & $-0.388 *$ & $-0.388 *$ & -0.503 & $-0.393^{*}$ & $-0.403^{*}$ & -0.386 & -0.305 & -0.707 \\
\hline FAST-THEN-SLOW & -0.193 & -0.192 & $-0.572 *$ & -0.175 & -0.275 & -0.413 & $-0.561^{*}$ & $-0.905^{*}$ \\
\hline SLOW-THEN-FAST & $-0.515 * *$ & $-0.514 * *$ & $-0.941 * *$ & $-0.504 * *$ & $-0.686 * *$ & $-0.811 * *$ & $-0.927 * *$ & $-1.598 * *$ \\
\hline Survey is long & & -0.004 & -0.495 & -0.016 & -0.064 & -0.201 & -0.366 & $-0.902 *$ \\
\hline Short invitation & & & & $-0.317 *$ & $-0.561 * *$ & $-0.539 * *$ & $-0.567 * *$ & $-0.529 * *$ \\
\hline 100 vs 50 points & & & & $0.412 * *$ & $0.432 * *$ & $0.531 * *$ & $0.450 * *$ & $0.559 * *$ \\
\hline 150 vs 50 points & & & & $0.470^{* *}$ & $0.393^{*}$ & $0.475^{*}$ & $0.380^{*}$ & $0.446^{*}$ \\
\hline Elapsed time (in min) & & & & & $0.208^{* *}$ & 0.026 & $0.159^{*}$ & -0.005 \\
\hline Elapsed time squared (in min) & & & & & $-0.001 * *$ & $-0.007^{*}$ & $-0.001 * *$ & $-0.008^{*}$ \\
\hline Age & & & & & $-0.018^{* *}$ & -0.008 & $-0.018 * *$ & -0.009 \\
\hline Log of experience & & & & & $2.065^{* *}$ & $2.098 * *$ & $1.975^{* *}$ & $2.071 * *$ \\
\hline Checkpoint trend & & & & & $-0.171 * *$ & -0.006 & $-0.168 * *$ & -0.031 \\
\hline \multicolumn{9}{|l|}{ Interactions with elapsed time } \\
\hline LINEAR INDICATOR & & & & & & & 0.170 & 0.110 \\
\hline FAST-THEN-SLOW & & & & & & & -0.021 & -0.018 \\
\hline SLOW-THEN-FAST & & & & & & & 0.056 & 0.029 \\
\hline \multicolumn{9}{|c|}{ Interactions with checkpoint trend } \\
\hline LINEAR INDICATOR & & & & & & & -0.028 & -0.036 \\
\hline FAST-THEN-SLOW & & & & & & & 0.007 & 0.056 \\
\hline SLOW-THEN-FAST & & & & & & & -0.001 & 0.063 \\
\hline \multicolumn{9}{|l|}{ Interactions with survey is long } \\
\hline LINEAR INDICATOR & & & 0.246 & & & & 0.109 & 0.469 \\
\hline FAST-THEN-SLOW & & & 0.726 & & & & 0.382 & 0.639 \\
\hline SLOW-THEN-FAST & & & $0.844 *$ & & & & 0.620 & $1.270 * *$ \\
\hline \multicolumn{9}{|l|}{ Interaction with experienced } \\
\hline LINEAR INDICATOR & & & & & & & 0.372 & -0.024 \\
\hline FAST-THEN-SLOW & & & & & & & 0.388 & 0.241 \\
\hline SLOW-THEN-FAST & & & & & & & -0.048 & -0.127 \\
\hline Constant & $5.669^{* *}$ & $5.670 * *$ & $5.914 * *$ & $5.890 * *$ & $5.428^{* *}$ & $3.457 * *$ & $6.000 * *$ & $4.563 * *$ \\
\hline Observations & 57601 & 57601 & 57601 & 57601 & 55389 & 43130 & 55389 & 43130 \\
\hline Pseudo $R^{2}$ & 0.003 & 0.003 & 0.005 & 0.008 & 0.209 & 0.139 & 0.213 & 0.146 \\
\hline
\end{tabular}

Robust $p$ values in parentheses. *significant at $5 \%$, **significant at $1 \%$.

reached the previous one. For this we create a new data set, generating as many rows in the data per respondent as the number of checkpoints that the respondent has passed, plus one extra row for the checkpoint that he or she did not pass. Because we have multiple data rows per respondent, we adapt the standard errors of our estimates using the method of Huber (1967). For an introduction and overview see Elandt-Johnson and Johnson (1999) or Hosmer and Lemeshow (1999). Here it should be stressed that the conversion of the data to this so-called "person-period format" is a standard procedure in survival analysis (in this case generating about 55,000 quasi-cases).

Table 5 shows the results of our analyses. In the first column we present the analysis with only dummy-variables that represent the different indicators. The reference category is
NO INDICATOR. There is a negative effect of the progress indicator for all three types of indicators, with both the effects of the LINEAR INDICATOR and the SLOW-THEN-FAST indicator statistically significantly different from zero (at the $p=.036$ and $p=.004$ level, respectively).

According to Hypothesis 2, the effects of the indicators should vary with the questionnaire length. The second and third models in Table 5 show the results when the variable long survey and its interaction with the different progress indicators are added. There was no significant main effect of the length of the survey, but the effect of the SLOWTHEN-FAST indicator was less negative for the survival in the longer survey $(\mathrm{b}=0.84, p=.02)$, providing some support for Hypothesis 2.

Several other factors were taken into account, some of 
Table 6. Logistic regression analyses on the probability that the respondent allows additional questions (first three models) and regression analysis on "user satisfaction" (fourth model); coefficients are unstandardized

\begin{tabular}{llccc}
\hline & Allowed extra questions & Allowed extra questions & Allowed extra questions & User satisfaction \\
\hline LINEAR INDICATOR & 0.158 & 0.155 & 0.197 & 0.016 \\
FAST-THEN-SLOW & $0.297^{*}$ & $0.297^{*}$ & 0.296 & -0.049 \\
SLOW-THEN-FAST & $0.540^{* *}$ & $0.537^{* *}$ & $0.628^{* *}$ & -0.049 \\
Short invitation & & -0.008 & -0.017 & -0.019 \\
100 vs 50 points & & 0.148 & 0.136 & -0.041 \\
150 vs 50 points & & 0.110 & 0.071 & -0.037 \\
Survey is long & & 0.035 & 0.056 & -0.004 \\
Age & & -0.002 & $0.008^{* *}$ \\
Log of experience & & $0.516^{* *}$ & $0.124^{* *}$ \\
Elapsed time (min.) & & $0.341^{* *}$ & $0.122^{* *}$ \\
Elapsed time squared (min.) & & & $-0.006^{* *}$ & $-0.003^{* *}$ \\
Constant & $1.207^{* *}$ & $1.114^{* *}$ & $-3.037^{* *}$ & $-1.517^{* *}$ \\
Observations & 2197 & 2197 & 2197 & 1775 \\
Pseudo $R^{2}$ & 0.006 & 0.007 & 0.095 & 0.059 \\
$R^{2}$ & & & \\
\hline
\end{tabular}

$p$ values in parentheses. *significant at $5 \%, * *$ significant at $1 \%$.

which are not orthogonal to the progress-indicator dummies and which might influence the effects of the progress indicators on survey continuation. We included the following variables in Model 5 of Table 5: the elapsed time and its square, the age of the respondent, the survey experience of the respondent (log of experience), and the position in the survey (checkpoint trend). We controlled for effects of separate questions by including the different checkpoint dummies $\mathrm{ChP} 1 \ldots \mathrm{ChP} 25$. The results showed that the probability of continuing the survey decreased with age, $p=.001$, and strongly increased with experience, $p<.001$. Elapsed time was also controlled for, as the more checkpoints that have been reached, the more likely it is that people drop out, $p<.001$. This finding disappeared when what happens after Checkpoint 5 is taken into account. All other effects remain stable.

Model 6 of Table 5 shows what happens when only the behavior of those who have passed Checkpoint 4 is considered. Most effects remain stable, but time and checkpoint both change. The effect of checkpoint now disappears, $p=$ .824 , whereas the effect of time is now decreasing, providing no support for Hypothesis 3, which claimed an effect of the opposite direction.

In Model 7 and 8, interactions with the progress-indicator dummies are included, which finally allows a formal test of our main Hypothesis 1. Most interactions did not approach significance. The only interaction effects that showed significant differences at the $p=.10$ level are the interaction between length of the survey and the SLOWTHEN-FAST indicator $(p=.02, p=.09$, and $p=.008$ in Models 3, 7, and 8, respectively). A closer look indicates that the SLOW-THEN-FAST indicator performed significantly worse than no indicator for the short survey, and for the long survey the estimated coefficient is still negative but not statistically significant.
The nonexistence of statistically significant interaction effects between the type of progress indicator and the position in the survey is consistent with Hypothesis 1: The results support that the first impression that an indicator makes is crucial.

Two indicators were used to measure satisfaction and to test Hypothesis 4 about the respondent's survey satisfaction. At the end of our survey, satisfaction should be higher for the SLOW-THEN-FAST indicator than for the FAST-THENSLOW indicator. The first one is whether respondents were willing to answer some additional answers. Table 6 shows the results of a logistic regression analysis with this variable as the dependent variable in Models 1-3. Indeed, respondents in the SLOW-THEN-FAST condition had a higher probability of allowing a few extra questions ( $p=.001$, about 8 percentage points higher than without a progress indicator). The strange thing is that the respondents in the FAST-THENSLOW condition also seemed to have a higher probability of allowing some extra questions, though only at $p$ levels of around 0.05 . In any case, the difference between these two conditions was, as in Böhme (2003), not statistically significant $(p=.14)$, lending no support for Hypothesis 4 .

Model 4 in Table 6 shows the results of an analysis with the second indicator of user satisfaction. Older respondents were more satisfied $(p<.001)$, more experienced respondents were more satisfied $(p=.006)$, and those who used more time are more satisfied $(p<.001)$. None of the progress indicator variables approached significance. It appears that any differences in satisfaction that are caused by the kind of progress indicator are subsumed when we look only at the respondents who were willing to answer some extra questions. So there is some support for the hypothesis that respondents in the SLOW-THEN-FAST and the FAST-THENSLOW conditions were more satisfied toward the end of the 
Table 7. Regression analyses on the time it takes to complete the survey; coefficients are unstandardized

\begin{tabular}{|c|c|c|c|c|}
\hline & Time (min.) & Time (min.) & Time (min.) & Time (min.) \\
\hline LINEAR INDICATOR & -0.159 & -0.182 & -0.167 & -0.192 \\
\hline FAST-THEN-SLOW & 0.100 & 0.064 & 0.098 & 0.079 \\
\hline SLOW-THEN-FAST & -0.208 & -0.231 & -0.207 & -0.207 \\
\hline Short invitation & -0.233 & -0.226 & -0.228 & -0.228 \\
\hline 100 vs 50 points & 0.273 & 0.286 & 0.282 & 0.284 \\
\hline 150 vs 50 points & 0.187 & 0.206 & 0.192 & 0.188 \\
\hline Survey is long & -0.110 & -0.121 & -0.110 & -0.113 \\
\hline Age & $0.077 * *$ & $0.077 * *$ & $0.077 * *$ & $0.077 * *$ \\
\hline Experience & $-0.809 * *$ & $-0.800 * *$ & $-0.813 * *$ & $-1.110 * *$ \\
\hline Computer literacy (tasks) & $0.558^{*}$ & $0.577 *$ & 0.561 & $0.561 *$ \\
\hline Computer literacy (self) & $-0.824 * *$ & $-1.244 * *$ & $-0.817 * *$ & $-0.822 * *$ \\
\hline \multicolumn{5}{|c|}{ Interactions with computer literacy (self) } \\
\hline LINEAR INDICATOR & & 0.294 & & \\
\hline FAST-THEN-SLOW & & $1.212 * *$ & & \\
\hline SLOW-THEN-FAST & & 0.205 & & \\
\hline \multicolumn{5}{|c|}{ Interactions with computer literacy (tasks) } \\
\hline LINEAR INDICATOR & & & -0.413 & \\
\hline FAST-THEN-SLOW & & & 0.368 & \\
\hline SLOW-THEN-FAST & & & 0.024 & \\
\hline \multicolumn{5}{|l|}{ Interactions with experience } \\
\hline LINEAR INDICATOR & & & & 0.594 \\
\hline FAST-THEN-SLOW & & & & 0.497 \\
\hline SLOW-THEN-FAST & & & & 0.143 \\
\hline Constant & $16.772 * *$ & $18.541 * *$ & $16.733 * *$ & $17.284 * *$ \\
\hline Observations & 2183 & 2183 & 2183 & 2183 \\
\hline$R^{2}$ & 0.059 & 0.063 & 0.059 & 0.059 \\
\hline
\end{tabular}

$p$ values in parentheses. *significant at 5\%,**significant at $1 \%$.

survey, though precise reasons remain unclear. In any case, this does not support the implicit ideas of Hypotheses 1 and 4 that the differential behavior of the progress indicators at the end of the survey affects the satisfaction.

According to Hypothesis 5, one would expect an interaction effect between computer literacy and type of indicator on the needed response time. Table 7 shows that Hypothesis 5 is not supported. In fact, if anything, the results show that it is the other way around. When we consider the self-reported computer literacy variable (Models 1 and 2 in Table 7), the effect of computer literacy disappears in the FAST-THEN-SLOW condition. According to Hypothesis 5 , however, the effect should be stronger for this indicator. We have no explanation for this unexpected finding. Including or excluding other independent factors or interaction effects did not change the results substantially.

\section{Conclusion and Discussion}

The study analyzed the effects of three kinds of progress indicators on survey dropout and survey satisfaction: lin- ear progress indicator, a fast-then-slow progress indicator, and a slow-then-fast progress indicator. The behavior of respondents in a web survey with the three indicators was compared with the behavior of respondents in the same survey without any indicator. We clarify two theoretical expectations concerning how the indicators should affect the tendency to drop out, namely via its first impression at the beginning of a survey or via a surfacing effect that depends on the respondent's position in the questionnaire. Contrary to other studies that only analyzed the completion rates, in our study we, therefore, tracked respondent behavior throughout the whole survey and applied survival analysis to analyze this behavior. In addition, we analyzed whether the effects depend on the questionnaire length.

The results do not provide any evidence for the hypothesis that the effects of the progress indicators on the tendency to continue participating in the survey depend on the respondent's position in the questionnaire. When indicators had an effect, they started to affect the tendency to continue participating in the first part of the questionnaire and these effects seemed to persist throughout the questionnaire irrespective of the behavior of the progress indicator. Our results, therefore, support the hypothesis that first impres- 
sions matter and, moreover, emphasize that this is the first time that the hypothesis has been tested adequately (through survival analysis).

Contrary to some of the expectations in the literature, the main effects of the indicators tend to be negative. That is, adding a progress indicator did not increase and sometimes - depending on the questionnaire length - decreased the participation rates of respondents. For instance, in the shorter questionnaire, the SLOW-THEN-FAST indicator decreased the probability of continuing with the survey when compared to a respondent who did not see any indicator. We assume that in these cases the indicator draws the respondent's attention to the fact that the end of the survey is far away, which then increases the probability of stopping participation. The only beneficial effects that we found were that respondents who were confronted with a SLOW-THEN-FAST or a FAST-THEN-SLOW indicator were more willing to answer some additional questions than respondents who were not confronted with an indicator. However, the reasons for the differences remain unclear: We found no difference for the linear indicator. Perhaps beneficial effects are triggered if the respondent has at least, at some time in the survey, experienced a progress indicator that progresses fast, but several other explanations are possible and beyond the realm of this study. The most important implication of this study for the design of web surveys is that survey designers should be reluctant about including any kind of progress indicator for surveys that take about $20 \mathrm{~min}$ or more.

Some aspects of our study might limit the scope of its conclusions. The survey was rather long; in the "shorter" version it still took about $20 \mathrm{~min}$ to answer. In addition, the questionnaire itself was relatively heterogeneous. It included topics ranging from behavior in online auctions to opinions about Dutch politics. Moreover, the respondents were sampled from an online panel. Results might be different for specific target groups surveyed on a single topic. We have no strong hypotheses as to the direction of the differences with our study but we can imagine that panelists, who after all opt to receive surveys voluntarily, are more likely to complete the survey, so that the differences between progress indicators are likely to be more pronounced in nonpanel samples.

Two methodological contributions with future implications should be emphasized. First, in this kind of research it is sensible to use survival analysis as the standard technique for testing the effects of design and layout issues on survey compliance. Second, it is important to observe that relatively large samples (typically more than 1000 respondents) are necessary for drawing conclusions about effects on survey compliance.

There are other opportunities to continue with this line of research to find out whether progress indicators increase the respondents' tendency to continue with a web survey. First of all, as Hypothesis 2 suggests, progress indicators might stimulate more beneficial inferences of the respondent about the time burden under the condition of a shorter survey since then they do not draw the respondent's attention to the fact that the end of the survey is still so far away. Furthermore, it is worthwhile to change the degree of nonlinearity of the indicators. The nonlinear indicators we used were not very extreme and one might try more extreme versions. However, one should take care that providing such misleading information in itself goes against one of the reasons to provide the progress indicators in the first place (namely, to show care-intensity). Another problem to be solved is the avoidance of "jumps" of progress indicators that are induced by filter questions (Kaczmirek, 2008). Also, one could think of an indicator of the sort "X pages to go," or of a selective use of an indicator or of a combination of different indicators at different positions of the survey. In the light of our findings, a placement of indicators early in the questionnaire seems to be most promising.

\section{Acknowledgments}

We thank Lars Kaczmirek (ZUMA, Mannheim) and other participants at the 8th General Online Research conference (GOR'06) for useful suggestions and comments on an earlier version of this paper.

\section{References}

Bandilla, W., \& Bosnjak, M. (2000). Online-Surveys als Herausforderung für die Umfrageforschung [Online surveys as a challenge to survey research]. In P.P. Mohler \& P. Lüttinger. (Eds.), Festschrift für Max Kaase [Festschrift for Max Kaasse] (pp. 9-28). Mannheim: ZUMA:

Böhme, R. (2003). Fragebogeneffekte bei Online-Befragungen [Questionnaire effects in online surveys]. Unpublished Master Thesis, Technische Universität Dresden.

Bosnjak, M., \& Tuten, T.L. (2001). Classifying response behavior in web-based surveys. Journal of Computer-Mediated Communication, 6(3), 1-17.

Conrad, F., Couper, M., Tourangeau, R., \& Peytchev, A. (2005). Impact of progress feedback on task completion: First impressions matter. Paper presented at the conference CHI, Portland, Oregon, USA, April 2-7, 2005.

Couper, M. (2000). Usability evaluation of computer-assisted survey instruments. Social Science Computer Review, 4, 384-396.

Couper, M., Traugott, M.W., \& Lamias, M.J. (2001). Web survey design and administration. Public Opinion Quarterly, 65, 230-253.

Crawford, S.D., Couper, M., \& Lamias, M.J. (2001). Web surveys: Perception of burden. Social Science Computer Review, 19, 146-162.

Dillman, D.A. (2000). Mail and internet surveys. The tailored design method. New York, Chichester: Wiley.

Elandt-Johnson, R., \& Johnson, N. (1999). Survival models and data analysis. New York: Wiley.

Gräf, L. (2002). Assessing internet questionnaires: The online pretest lab. In B. Batinic, U.D. Reips, \& M. Bosnjak (Eds.), Online social sciences (pp. 49-68). Seattle: Hogrefe \& Huber. 
Heerwegh, D. (2004). Using progress indicators in web surveys. Paper prepared for the 59th AAPOR conference, Phoenix, Arizona, May 13-16, 2004.

Heerwegh, D., \& Loosveldt, G. (2006). An experimental study on the effects of personalization, survey length statements, progress indicators, and survey sponsor logos in Web Surveys. Journal of Official Statistics, 22, 191-210.

Hosmer, D.W., \& Lemeshow, S. (1999). Applied survival analysis: Regression modeling of time to event data. New York: Wiley.

Huber, P.J. (1967). The behavior of maximum likelihood estimates under nonstandard conditions. Proceedings of the Fifth Berkeley Symposium on Mathematical Statistics and Probability, 1, 221-233.

Kaczmirek, L. (2008). Human-survey interaction: Usability and nonresponse in online surveys. University of Mannheim.

Lin, N., \& Dumin, M. (1986). Access to occupations through social ties. Social Networks, 8, 365-385.

Schwarz, N. (1998). Cognition and communication. Mahwah, NJ: Erlbaum.

Smyth, J.D., Dillman, D.A., Christian, L.M., \& Stern, M.J. (2006). Effects of using visual design principles to group re- sponse options in web surveys. International Journal of Internet Science, 1, 6-16.

Tourangeau, R., Rips, L.J., \& Rasinski, K. (2007). The psychology of survey response. Cambridge, UK: Cambridge University Press.

Received July 7, 2008

Final revision received September 2, 2008

Accepted September 3, 2008

Uwe Matzat

Sociology Section

Department of Technology Management

Eindhoven University of Technology

P.O. Box 513

NL-5600 MB Eindhoven

The Netherlands

Tel. +31 40 247-8392

E-mail u.matzat@tm.tue.nl 